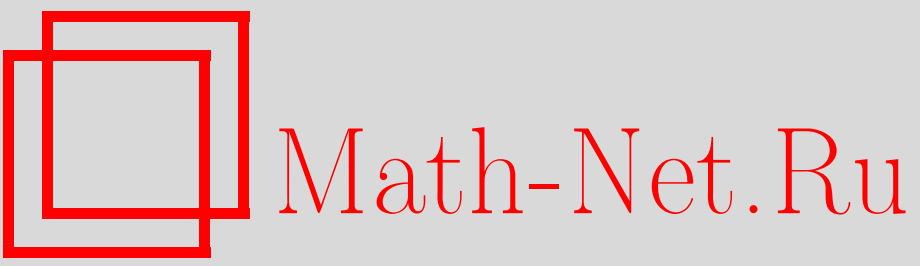

А. И. Ахиезер, Л. Н. Давыдов, С. В. Пелетминский, К кинетической теории магнитной гидродинамики нейтронной материи, ТМФ, 2000, том 125, номер 3, 471490

DOI: https://doi.org/10.4213/tmf680

Использование Общероссийского математического портала Math-Net.Ru подразумевает, что вы прочитали и согласны с пользовательским соглашением http://www.mathnet.ru/rus/agreement

Параметры загрузки:

IP: 3.95 .254 .165

26 апреля 2023 г., 15:08:36 
ТЕОРЕТИЧЕСКАЯ

И МАТЕМАТИЧЕСКАЯ

ФИЗИКА

Том 125, 3

декабрь, 2000

(c) 2000 г. А.И. Ахиезер, Л.Н. Давыдов* , С. В. Пелетминский*

\section{К КИНЕТИЧЕСКОЙ ТЕОРИИ МАГНИТНОЙ ГИДРОДИНАМИКИ НЕЙТРОННОЙ МАТЕРИИ}

В микроскопическом подходе, основанном на теории ферми-жидкости Ландау, выведены уравнения магнитной гидродинамики нейтронной материи, в которых, наряду с сильными короткодействующими ядерными взаимодействиями, учитываются слабые дальнодействующие магнитные взаимодействия. Рассмотрены приложения полученных уравнений магнитной гидродинамики к теории ударных волн в нейтронной материи.

\section{1. ВВЕ ДЕНИЕ}

В работе [1] на основе феноменологического подхода были построены уравнения магнитной гидродинамики нейтронной материи, в которых, наряду с сильными короткодействуюшими ядерными взаимодействиями, учитывались слабые дальнодействующие магнитные взаимодействия. В данной работе на основе ферми-жидкостного подхода Ландау дан микроскопический вывод этих уравнений, а также рассмотрены приложения полученных уравнений магнитной гидродинамики к теории ударных волн в нейтронной материи.

Основополагаюшую роль в ферми-жидкостном подходе Ландау играет энергия системы, которая является функционалом одночастичной матрицы плотности квазичастиц. В работе сформулированы и широко используются свойства симметрии функционала энергии по отношению к преобразованиям пространственных трансляций и пространственных поворотов. Кроме того, важную роль при построении гидродинамики играет интеграл столкновений квазичастиц. В работе сформулированы наиболее общие свойства интеграла столкновений, приводящего в процессе эволюции к формированию квазиравновесного состояния квазичастиц, которое определяется не только пространственно-неоднородным распределением обычных гидродинамических параметров, но и пространственно-неоднородным распределением магнитных моментов.

На основе этого квазиравновесного состояния найдены выражения для плотностей потоков всех гидродинамических параметров с учетом слабых магнитных взаимодействий. Найдены ограничения, налагаемые на уравнения магнитной гидродинамики требованием галилеевой инвариантности теории.

${ }^{*}$ Национальный научный центр "Харьковский физико-технический институт”, Харьков, Украина 


\section{2. ОСНОВНЫЕ ПОЛОЖЕНИЯ ТЕОРИИ ФЕРМИ-ЖИДКОСТИ ЛАНДАУ}

Как известно, состояние ферми-жидкости характеризуется одночастичной матрицей плотности $f_{\kappa \kappa^{\prime}}$, где $\kappa=(\mathbf{p}, \sigma)$ - квантовые числа, описывающие состояние квазичастиц $(\mathbf{p}-$ импульс и $\sigma$ - проекция спина). Для описания состояния системы (как равновесного, так и неравновесного) необходимо задать энергию ферми-жидкости как функционал одночастичной матрицы плотности. Этот функционал играет роль гамильтониана в обычной микроскопической теории. Равновесное состояние ферми-жидкости соответствует максимуму комбинаторной энтропии

$$
S(\hat{f})=-\operatorname{Sp}[\hat{f} \ln \hat{f}+(1-\hat{f}) \ln (1-\hat{f})]
$$

при фиксированном функционале энергии $E(\hat{f})$ и фиксированных аддитивных интегралах движения $\gamma_{\alpha}(\hat{f})=\operatorname{Sp} \hat{f} \hat{\gamma}_{\alpha}$, где $\hat{\gamma}_{\alpha}$ - операторы аддитивных интегралов движения ( $\hat{\gamma}_{k}=\hat{p}_{k}(k=1,2,3)$ - оператор импульса, $\hat{\gamma}_{4}=1$ - оператор числа квазичастиц). Здесь $\hat{f}$ - статистический оператор, соответствующий матрице плотности $f_{\kappa \kappa^{\prime}}$. Задаче на условный максимум функционала $S(\hat{f})$ соответствует задача определения безусловного минимума функционала

$$
\Omega(\hat{f})=-S(\hat{f})+\beta E(\hat{f})+Y_{\alpha} \gamma_{\alpha}(\hat{f})
$$

где $\beta=T^{-1}$ и $Y_{\alpha}$ - множители Лагранжа ( $T$ - температура, $\mu=-Y_{4} / \beta$ - химический потенциал, $v_{k}=-Y_{k} / \beta(k=1,2,3)-$ скорость $)$. Решение этой задачи дается выражением

$$
\hat{f}=\left[\exp \left[\beta \hat{\varepsilon}(\hat{f})+Y_{\alpha} \hat{\gamma}_{\alpha}(\hat{f})\right]+1\right]^{-1}
$$

где матричный элемент оператора $\hat{\varepsilon}(\hat{f})$ определяется формулой

$$
\varepsilon_{\kappa \kappa^{\prime}}=\frac{\partial E(\hat{f})}{\partial f_{\kappa \kappa^{\prime}}} .
$$

Для описания неравновесных процессов в ферми-жидкости в приближении $\omega \tau \gg 1$ ( $\omega$ - характерная частота исследуемых процессов, $\tau$ - время релаксации), когда можно пренебречь интегралом столкновений, используется кинетическое уравнение

$$
i \frac{\partial \hat{f}}{\partial t}-[\hat{\varepsilon}(\hat{f}), \hat{f}]=0 .
$$

Для формулировки свойств симметрии функционала энергии по отношению к тем или иным преобразованиям введем функционал плотности энергии $E(\mathbf{x}, \hat{f})$, связанньй с функционалом энергии $E(\hat{f})$ ферми-жидкости формулой

$$
E(\hat{f})=\int d^{3} x E(\mathbf{x}, \hat{f}) .
$$

Будем говорить, что этот функционал инвариантен по отношению к преобразованию трансляции, если

$$
E(\mathbf{x}+\mathbf{y}, \hat{f})=E\left(\mathbf{x}, e^{i \hat{\mathbf{p}} \mathbf{y}} \hat{f} e^{-i \hat{\mathbf{p}} \mathbf{y}}\right),
$$


где $\mathbf{y}-$ произвольный вектор трансляции.

Функционал плотности энергии $E(\mathbf{x}, \hat{f})$ будет инвариантен относительно пространственных поворотов, если выполняется следуюшее соотношение:

$$
E(\mathbf{x}, \hat{f})=E\left(\hat{a} \mathbf{x}, U^{+}(a) \hat{f} U(a)\right),
$$

где $\hat{a}$ - матрица ортогональных поворотов и унитарный оператор $U(a)$ определяется из соотношения $U \hat{x}_{i} U^{+}=a_{i k} \hat{x}_{k}\left(\hat{x}_{i}\right.$ - оператор координаты). В случае бесконечно малых поворотов

$$
a_{i k}=\delta_{i k}+\varepsilon_{i k}, \quad U(a)=1-\frac{i}{2} \varepsilon_{i k} J_{i k},
$$

где $J_{i k}-$ полный момент количества движения квазичастицы,

$$
\hat{J}_{i k}=\varepsilon_{i k l} \hat{J}_{l}, \quad \hat{J}_{l}=\hat{L}_{l}+\widehat{S}_{l} .
$$

Здесь $\hat{\mathbf{L}}=\hat{\mathbf{x}} \times \hat{\mathbf{p}}-$ орбитальный момент количества движения, $\widehat{\mathbf{S}}-$ спиновый момент количества движения квазичастищы, $\varepsilon_{i k l}$ - единичный антисимметричный тензор.

Перечисленные свойства симметрии функционала энергии всегда выполняются в отсутствие внешних полей, т.к. они связаны со свойствами симметрии пространства по отношению к трансляциям и поворотам. Однако функционал энергии в пренебрежении обменными релятивистскими взаимодействиями (такими, как спин-орбитальное и магнитодипольное взаимодействия) по сравнению с ядерными взаимодействиями может обладать дополнительными свойствами симметрии, связанными, например, с инвариантностью относительно поворотов спинов (спиновая инвариантность),

$$
E(\mathbf{x}, \hat{f})=E\left(\mathbf{x}, e^{i \omega_{i} S_{i}} \hat{f} e^{-i \omega_{i} S_{i}}\right)
$$

где $\omega_{i}-$ произвольньй вектор поворота спинов.

Введем оператор плотности энергии квазичастицы

$$
\varepsilon_{\kappa \kappa^{\prime}}(\mathbf{x}, \hat{f})=\frac{\partial E(\mathbf{x}, \hat{f})}{\partial f_{\kappa^{\prime} \kappa}} .
$$

Эта величина связана с оператором энергии квазичастицы $\hat{\varepsilon}(\hat{f})$ соотношением

$$
\hat{\varepsilon}(\hat{f})=\int d^{3} x \hat{\varepsilon}(\mathbf{x}, \hat{f}) .
$$

С помошью введенной величины $\hat{\varepsilon}(\mathbf{x}, \hat{f})$ легко сформулировать инфинитезимальные свойства рассмотренных симметрий. Так, в случае пространственных трансляций согласно (2.7) имеем

$$
i \operatorname{Sp} \hat{f}\left[\hat{\varepsilon}(\mathbf{x}, \hat{f}), p_{k}\right]=\frac{\partial E(\mathbf{x})}{\partial x_{k}} .
$$

В случае пространственных врашений согласно (2.8)

$$
i \operatorname{Sp} \hat{f}\left[\hat{\varepsilon}(\mathbf{x}, \hat{f}), J_{i}\right]=\varepsilon_{i k l} x_{k} \frac{\partial E(\mathbf{x})}{\partial x_{l}} .
$$

Если пренебречь релятивистским взаимодействием, то в соответствии с (2.10) имеем

$$
i \operatorname{Sp} \hat{f}\left[\hat{\varepsilon}(\mathbf{x}, \hat{f}), S_{i}\right]=0 .
$$


Кроме того, варьируя соотношения $(2.7)$ и $(2.10)$ по матрище плотности $f_{\kappa \kappa^{\prime}}$, получим в случае пространственных трансляций

$$
e^{-i \hat{\mathbf{p}} \mathbf{y}} \varepsilon\left(\mathbf{x}, e^{i \hat{\mathbf{p}} \mathbf{y}} \hat{f} e^{-i \hat{\mathbf{p}} \mathbf{y}}\right) e^{i \hat{\mathbf{p}} \mathbf{y}}=\varepsilon(\mathbf{x}+\mathbf{y}, \hat{f})
$$

и в случае спиновых врашений

$$
e^{-i \omega_{i} \widehat{S}_{i}} \varepsilon\left(\mathbf{x}, e^{i \omega_{i} \widehat{S}_{i}} \hat{f} e^{-i \omega_{i} \widehat{S}_{i}}\right) e^{i \omega_{i} \widehat{S}_{i}}=\varepsilon(\mathbf{x}, \hat{f}) .
$$

Подчеркнем, что соотношения (2.13)- (2.17) должны выполняться для произвольного статистического оператора $\hat{f}$. Эти соотношения будут нами использованы в дальнейшем для получения плотностей потоков аддитивных интегралов движения и соответствуюших им законов сохранения, связанных с указанными свойствами симметрии.

\section{3. УРАВНЕНИЯ МАГНИТНОЙ ГИДРОДИНАМИКИ НЕЙТРОННОЙ МАТЕРИИ}

Мы в дальнейшем будем интересоваться низкочастотными явлениями в фермижидкости, когда $\omega \tau \ll 1$. В этом случае неприменимо кинетическое уравнение $(2.5)$, которое справедливо в области $\omega \tau \gg 1$, и необходимо учитывать столкновения между квазичастицами, т.е. дополнить уравнение (2.5) интегралом столкновений $I(\hat{f})[2]$. Поэтому кинетическое уравнение примет вид

$$
\frac{\partial \hat{f}}{\partial t}+i\left[\hat{\varepsilon}(\hat{f})+\hat{\varepsilon}^{\prime}(\hat{f}), \hat{f}\right]=I(\hat{f}),
$$

где $\hat{\varepsilon}(\hat{f})$ - энергия квазичастицы, обусловленная ядерными взаимодействиями (которые мы будем предполагать не зависяшими от спинов), а $\hat{\varepsilon}^{\prime}(\hat{f})$ - более слабыми магнитными взаимодействиями. Интеграл столкновений $I(\hat{f})$ обусловлен только сильным ядерным взаимодействием.

В дальнейшем мы не будем интересоваться диссипативными гидродинамическими процессами, поэтому для получения уравнений идеальной гидродинамики не нужна явная структура интеграла столкновений, а будут необходимы только его некоторые общие свойства. Так же как и в случае интеграла столкновений Больцмана, взаимодействие в процессе столкновений носит локальньй характер, в силу чего среднее значение плотности аддитивных интегралов движения $\hat{a}(\mathbf{x})$ и плотности энергии квазичастищ $\hat{\varepsilon}(\mathbf{x}, \hat{f})$ не будут меняться в процессе столкновений, т.е.

$$
\operatorname{Sp} \hat{a}(\mathbf{x}) I(\hat{f})=0, \quad \operatorname{Sp} \hat{\varepsilon}(\mathbf{x}, \hat{f}) I(\hat{f})=0,
$$

где величины $\hat{a}(\mathbf{x})$ означают либо оператор плотности числа квазичастиц

$$
\hat{\rho}(\mathbf{x})=\delta(\mathbf{x}-\hat{\mathbf{x}})
$$

( $\hat{\mathbf{x}}$ - оператор координаты), либо оператор плотности импульса

$$
\hat{\pi}_{i}(\mathbf{x})=\frac{1}{2}\left\{\hat{p}_{i}, \delta(\mathbf{x}-\hat{\mathbf{x}})\right\}
$$


$\left(\hat{p}_{i}-\right.$ оператор импульса), либо оператор плотности спина

$$
\hat{s}_{i}(\mathbf{x})=\widehat{S}_{i} \delta(\mathbf{x}-\hat{\mathbf{x}})
$$

$\left(\widehat{S}_{i}\right.$ - оператор спина).

Сделаем несколько обших замечаний об используемых нами понятиях. Теория фермижидкости была бы неполной, если бы в ней, наряду с одночастичным статистическим оператором $\hat{f}$, не вводились вполне определенные операторы различных физических величин. Как известно, состояние ферми-жидкости описывается одночастичной матрицей плотности $f_{p_{1}, p_{2}} \equiv\left\langle\mathbf{p}_{1}|\hat{f}| \mathbf{p}_{2}\right\rangle$, где $|\mathbf{p}\rangle$ - собственный вектор квазичастицы с определенным импульсом $\hat{p}_{i}|\mathbf{p}\rangle=p_{i}|\mathbf{p}\rangle$ (здесь для простоты мы опускаем индекс спина) и $\hat{f}$ - одночастичный статистический оператор (не зависяший от выбора квантово-механического представления). Наряду с матрицей плотности в импульсном представлении часто используется вигнеровская функция распределения в фазовом пространстве

$$
f(\mathbf{x}, \mathbf{p})=\frac{1}{V} \sum_{\mathbf{p}^{\prime}} e^{-i \mathbf{p}^{\prime} \mathbf{x}}\left\langle\mathbf{p}+\frac{\mathbf{p}^{\prime}}{2}|\hat{f}| \mathbf{p}-\frac{\mathbf{p}^{\prime}}{2}\right\rangle,
$$

причем величина $f(\mathbf{x}, \mathbf{p})$ интерпретируется как “функция распределения" квазичастиц в фазовом пространстве $(\mathbf{x}, \mathbf{p})$, а величина $\int f(\mathbf{x}, \mathbf{p}) d \mathbf{p}-$ как плотность квазичастиц. Оператор координаты квазичастицы мы определяем формулой $\left[\hat{p}_{i}, \hat{x}_{i}\right]=-i \delta_{i k}$. Тогда собственный вектор оператора координаты $\hat{x}_{i}|\mathbf{x}\rangle=x_{i}|\mathbf{x}\rangle$ связан с собственным вектором $|\mathbf{p}\rangle$ оператора импульса соотношением

$$
|\mathbf{x}\rangle=\frac{1}{\sqrt{V}} \sum_{\mathbf{p}} e^{-i \mathbf{p x}}|\mathbf{p}\rangle .
$$

Используя эту формулу и формулу (3.6), легко найти, что

$$
\int f(\mathbf{x}, \mathbf{p}) d \mathbf{p}=\langle\mathbf{x}|\hat{f}| \mathbf{x}\rangle, \quad \int f(\mathbf{x}, \mathbf{p}) d \mathbf{x}=\langle\mathbf{p}|\hat{f}| \mathbf{p}\rangle
$$

или

$$
\int f(\mathbf{x}, \mathbf{p}) d \mathbf{x}=\operatorname{Sp} \hat{f} \delta(\mathbf{x}-\hat{\mathbf{x}}) .
$$

Так как в полуфеноменологической теории ферми-жидкости Ландау левая часть последнего равенства трактуется как плотность числа квазичастиц, то операторы $\hat{\mathbf{x}}$ и $\delta(\mathbf{x}-\hat{\mathbf{x}})$ должны трактоваться как оператор координаты и оператор плотности числа квазичастиц, соответственно.

Подчеркнем, что соотношения (3.2) справедливы при произвольном статистическом операторе $\hat{f}$. Кроме того, мы будем предполагать, что интеграл столкновений обрашается в нуль:

$$
I(\hat{f})=0,
$$

для квазиравновесного статистического оператора. Последний определяется как решение функционального уравнения, в котором учтены только ядерные взаимодействия

$$
\hat{f}=\{\exp \beta[\hat{\varepsilon}(\hat{f})-\mu-\hat{\mathbf{p}} \mathbf{v}-\widehat{\mathbf{m}} \tilde{\mathbf{h}}]+1\}^{-1}, \quad \widehat{m}_{i}=\mu_{f} \widehat{S}_{i}
$$


где $\mathbf{v}$ - скорость ферми-жидкости и $\tilde{\mathbf{h}}$ - термодинамическая переменная, определяющая магнитный момент $\mathbf{m}$ аналогично тому, как $\beta$ и $\mu$ определяют энергию и число квазичастиц ферми-жидкости. В отличие от равновесной матрицы плотности (2.3), матрица плотности (3.8) является квазиравновесной и соответствует равновесию, обусловленному только ядерными взаимодействиями. Благодаря этому, кроме обычных интегралов движения, в (3.8) появляется новый интеграл движения, связанный со спином. Вообще говоря, если бы квазичастицы обладали спином $s$, то появилось бы $r=(2 s+1)^{2}$ дополнительных (кроме энергии и импульса) интегралов движения. При $s=1 / 2$ имеем $r=4$ интеграла движения. Это единичный оператор в спиновом пространстве $\hat{I}$, соответствуюший термодинамической силе $\mu$, и три оператора проекций спина $\hat{s}_{i}$, соответствующих силе $\tilde{\mathbf{h}}$ (для бозонов при $s=1$ имели бы $r=9$ интегралов движения. Кроме $\hat{I}$ и $\hat{s}_{i}$ возникает пять интегралов движения, связанных с квадрупольным моментом $\left.\hat{s}_{i} \hat{s}_{k}+\hat{s}_{k} \hat{s}_{i}-(2 / 3) \delta_{i k} s(s+1)\right)$. Мы будем рассматривать фермионы со спином $s=1 / 2$. Поэтому вместо термина "квазичастица" будем иногда пользоваться термином “ферми$\mathrm{OH}$ ".

В соответствии со сказанным в начале этого раздела, представим плотность энергии ферми-жидкости в виде

$$
E_{t}(\mathbf{x}, \hat{f})=E(\mathbf{x}, \hat{f})+E^{\prime}(\mathbf{x}, \hat{f})
$$

где $E(\mathbf{x}, \hat{f})$ - плотность энергии ядерных взаимодействий и

$$
E^{\prime}(\mathbf{x}, \hat{f})=\frac{\mathbf{h}^{2}}{8 \pi}+\frac{1}{2} \alpha_{i k} \frac{\partial \mathbf{m}}{\partial x_{i}} \frac{\partial \mathbf{m}}{\partial x_{k}}+\frac{1}{2} R\left(m^{2}\right)-\mathbf{m H}_{0}
$$

- плотность энергии, обусловленная магнитодипольным взаимодействием, неоднородным и однородным обменными взаимодействиями, а также взаимодействием фермижидкости с внешним магнитным полем $\mathbf{H}_{0}$ [3]. В формуле (3.10)

$$
\mathbf{m}(\mathbf{x}, \hat{f})=\operatorname{Sp} \hat{f} \widehat{\mathbf{m}}(\mathbf{x}), \quad \widehat{\mathbf{m}}(\mathbf{x})=\mu_{f} \hat{\mathbf{s}}(\mathbf{x}),
$$

$\alpha_{i k}$ - тензор обменных постоянных и $\mathbf{h}(\mathbf{x})$ - магнитное поле, обусловленное сушествованием магнитного момента и определяемое уравнениями магнитостатики

$$
\operatorname{rot} \mathbf{h}=0, \quad \operatorname{div}(\mathbf{h}+4 \pi \mathbf{m})=0 .
$$

Последние уравнения можно переписать в виде

$$
\mathbf{h}=\nabla \varphi, \quad \operatorname{div} \mathbf{b}=0, \quad \mathbf{b}=\mathbf{h}+4 \pi \mathbf{m},
$$

откуда следует, что

$$
\delta \int d^{3} x \frac{h^{2}}{8 \pi}=\int d^{3} x \frac{1}{4 \pi} \mathbf{h}(\delta \mathbf{b}-4 \pi \delta \mathbf{m})=-\int d^{3} x \mathbf{h} \delta \mathbf{m}(\mathbf{x}, t) .
$$

Поэтому из формул (3.9), (3.10) вытекает соотношение

$$
\hat{\varepsilon}^{\prime}(\hat{f})=-\int d^{3} x \widehat{\mathbf{m}}(\mathbf{x}) \mathbf{h}^{\mathrm{eff}}, \quad \mathbf{h}^{\mathrm{eff}}=\mathbf{H}_{0}+\mathbf{h}+\alpha_{i k} \frac{\partial^{2} \mathbf{m}}{\partial x_{i} \partial x_{k}}-R\left(m^{2}\right) \mathbf{m} .
$$

Из кинетического уравнения (3.1) и формул (3.2) следует, что

$$
\frac{\partial \zeta_{\alpha}(\mathbf{x})}{\partial t}=i \operatorname{Sp} \hat{f}\left[\hat{\varepsilon}(\hat{f})+\hat{\varepsilon}^{\prime}(\hat{f}), \hat{\zeta}_{\alpha}(\mathbf{x})\right]
$$


где величины $\zeta_{\alpha}(\mathbf{x})$ соответствуют либо плотности $\hat{\rho}(\mathbf{x})$, либо плотности импульса $\hat{\pi}_{i}(\mathbf{x})$, либо плотности магнитного момента $\hat{s}_{i}(\mathbf{x})$. Замечая, что коммутатор $[\widehat{\mathbf{m}}(\mathbf{x}), \hat{\rho}(\mathbf{x})]=0$, имеем

$$
\frac{\partial \hat{\rho}(\mathbf{x})}{\partial t}=i \operatorname{Sp} \hat{f}[\hat{\varepsilon}(\hat{f}), \hat{\rho}(\mathbf{x})]
$$

Учитывая, что

$$
\left[\widehat{m}_{i}\left(\mathbf{x}^{\prime}\right), \hat{\pi}_{k}(\mathbf{x})\right]=i \widehat{m}_{i}(\mathbf{x}) \frac{\partial \delta\left(\mathbf{x}-\mathbf{x}^{\prime}\right)}{\partial x_{k}},
$$

получаем из $(3.15)$ при $\hat{\zeta}_{\alpha}(\mathbf{x})=\hat{\pi}_{k}(\mathbf{x})$ формулу

$$
\frac{\partial \pi_{k}(\mathbf{x})}{\partial t}=i \operatorname{Sp} \hat{f}\left[\hat{\varepsilon}(\hat{f}), \hat{\pi}_{k}(\mathbf{x})\right]+\mathbf{m}(\mathbf{x}) \frac{\partial \mathbf{h}^{\mathrm{eff}}(x)}{\partial x_{k}} .
$$

Полагая далее в $(3.15) \hat{\zeta}_{\alpha}(\mathbf{x})=\widehat{m}_{k}(\mathbf{x})$ и учитывая, что

$$
\left[\widehat{m}_{i}\left(\mathbf{x}^{\prime}\right), \widehat{m}_{k}(\mathbf{x})\right]=\mu_{f} \delta\left(\mathbf{x}-\mathbf{x}^{\prime}\right) \varepsilon_{i k l} \widehat{m}_{l}(\mathbf{x}),
$$

имеем

$$
\frac{\partial m_{k}(\mathbf{x})}{\partial t}=i \operatorname{Sp} \hat{f}\left[\hat{\varepsilon}(\hat{f}), \widehat{m}_{k}(\mathbf{x})\right]+\mu_{f}\left[\mathbf{m}(\mathbf{x}) \times \mathbf{h}^{\mathrm{eff}}(x)\right]_{k} .
$$

Приведем теперь уравнения (3.16)-(3.18) к виду дифференциальных законов сохранения. Замечая, что

$$
\begin{aligned}
i \operatorname{Sp} \hat{f}\left[\hat{\varepsilon}(\hat{f}), \hat{\zeta}_{\alpha}(\mathbf{x})\right]= & i \operatorname{Sp} \hat{f}\left[\hat{\varepsilon}(\mathbf{x}, \hat{f}), \int d^{3} x \hat{\zeta}_{\alpha}(\mathbf{x})\right]- \\
& -i \frac{\partial}{\partial x_{k}} \int d^{3} x^{\prime} x_{k}^{\prime} \int_{0}^{1} d \xi \operatorname{Sp} \hat{f}\left[\hat{\varepsilon}\left(\mathbf{x}-(1-\xi) \mathbf{x}^{\prime}, \hat{f}\right), \hat{\zeta}_{\alpha}\left(\mathbf{x}+\xi \mathbf{x}^{\prime}\right)\right],
\end{aligned}
$$

и используя формулы

$$
\begin{aligned}
i \operatorname{Sp} \hat{f}\left[\hat{\varepsilon}(\mathbf{x}, \hat{f}), \int d^{3} x \hat{\rho}(\mathbf{x})\right] & =0, \\
i \operatorname{Sp} \hat{f}\left[\hat{\varepsilon}(\mathbf{x}, \hat{f}), \int d^{3} x \hat{\pi}_{k}(\mathbf{x})\right] & =i \operatorname{Sp} \hat{f}\left[\hat{\varepsilon}(\mathbf{x}, \hat{f}), \hat{p}_{k}\right]=\frac{\partial E(\mathbf{x}, \hat{f})}{\partial x_{k}}, \\
i \operatorname{Sp} \hat{f}\left[\hat{\varepsilon}(\mathbf{x}, \hat{f}), \int d^{3} x \widehat{m}_{k}(\mathbf{x})\right] & =0,
\end{aligned}
$$

выражающие свойства симметрии функционала ядерной энергии ферми-жидкости по отношению к пространственным трансляциям и спиновым поворотам (см. (2.13)-(2.15)), получим

$$
\begin{aligned}
i \operatorname{Sp} \hat{f}[\hat{\varepsilon}(\hat{f}), \hat{\rho}(\mathbf{x})] & =-\frac{\partial j_{l}(\mathbf{x})}{\partial x_{l}}, \\
i \operatorname{Sp} \hat{f}\left[\hat{\varepsilon}(\hat{f}), \hat{\pi}_{k}(\mathbf{x})\right] & =-\frac{\partial t_{k l}(x)}{\partial x_{l}}, \\
i \operatorname{Sp} \hat{f}\left[\hat{\varepsilon}(\hat{f}), \hat{m}_{k}(\mathbf{x})\right] & =-\frac{\partial m_{k l}(\mathbf{x})}{\partial x_{l}},
\end{aligned}
$$


где

$$
\begin{gathered}
j_{k}(\mathbf{x})=i \int d^{3} x^{\prime} x_{k}^{\prime} \int_{0}^{1} d \xi \operatorname{Sp} \hat{f}\left[\hat{\varepsilon}\left(\mathbf{x}-(1-\xi) \mathbf{x}^{\prime}, \hat{f}\right), \hat{\rho}\left(\mathbf{x}+\xi \mathbf{x}^{\prime}\right)\right] \\
t_{k l}(\mathbf{x})=-E(\mathbf{x}, \hat{f}) \delta_{k l}+i \int d^{3} x^{\prime} x_{l}^{\prime} \int_{0}^{1} d \xi \operatorname{Sp} \hat{f}\left[\hat{\varepsilon}\left(\mathbf{x}-(1-\xi) \mathbf{x}^{\prime}, \hat{f}\right), \hat{\pi}_{k}\left(\mathbf{x}+\xi \mathbf{x}^{\prime}\right)\right] \\
m_{k l}(\mathbf{x})=i \int d^{3} x^{\prime} x_{l}^{\prime} \int_{0}^{1} d \xi \operatorname{Sp} \hat{f}\left[\hat{\varepsilon}\left(\mathbf{x}-(1-\xi) \mathbf{x}^{\prime}, \hat{f}\right), \widehat{m}_{k}\left(\mathbf{x}+\xi \mathbf{x}^{\prime}\right)\right]
\end{gathered}
$$

Получим теперь уравнения для плотности энергии ферми-жидкости, обусловленной ядерным взаимодействием $E(\mathbf{x}, \hat{f})$. Согласно определению $(2.11)$ имеем

$$
\frac{\partial E(\mathbf{x}, \hat{f})}{\partial t}=\operatorname{Sp} \hat{\varepsilon}(\mathbf{x}, \hat{f}) \frac{\partial \hat{f}}{\partial t} .
$$

Замечая, далее, что $\operatorname{Sp} \hat{\varepsilon}(\mathbf{x}, \hat{f}) I(\hat{f})=0$, и используя кинетическое уравнение $(3.1)$, получаем

$$
\frac{\partial E(\mathbf{x})}{\partial t}=i \operatorname{Sp} \hat{f}[\hat{\varepsilon}(\hat{f}), \hat{\varepsilon}(\mathbf{x}, \hat{f})]-i \int d^{3} x^{\prime} \mathbf{h}^{\mathrm{eff}}\left(\mathbf{x}^{\prime}\right) \operatorname{Sp} \hat{f}\left[\widehat{\mathbf{m}}\left(\mathbf{x}^{\prime}\right), \hat{\varepsilon}(\mathbf{x}, \hat{f})\right] .
$$

Для вывода дифференциального закона сохранения энергии с источником, обусловленным магнитным взаимодействием, заметим, что

$$
\begin{aligned}
i \operatorname{Sp} \hat{f}[\hat{\varepsilon}(\hat{f}), \hat{\varepsilon}(\mathbf{x}, \hat{f})]=i \operatorname{Sp} \hat{f}[\hat{\varepsilon}(\mathbf{x}, \hat{f}), \hat{\varepsilon}(\hat{f})]- \\
\quad-i \frac{\partial}{\partial x_{k}} \int d^{3} x^{\prime} x_{k}^{\prime} \int_{0}^{1} d \xi \operatorname{Sp} \hat{f}\left[\hat{\varepsilon}\left(\mathbf{x}-(1-\xi) \mathbf{x}^{\prime}, \hat{f}\right), \hat{\varepsilon}\left(\mathbf{x}+\xi \mathbf{x}^{\prime}, \hat{f}\right)\right]
\end{aligned}
$$

откуда следует соотношение

$$
\begin{aligned}
& i \operatorname{Sp} \hat{f}[\hat{\varepsilon}(\hat{f}), \hat{\varepsilon}(\mathbf{x}, \hat{f})]= \\
& \quad=-\frac{i}{2} \frac{\partial}{\partial x_{k}} \int d^{3} x^{\prime} x_{k}^{\prime} \int_{0}^{1} d \xi \operatorname{Sp} \hat{f}\left[\hat{\varepsilon}\left(\mathbf{x}-(1-\xi) \mathbf{x}^{\prime}, \hat{f}\right), \hat{\varepsilon}\left(\mathbf{x}+\xi \mathbf{x}^{\prime}, \hat{f}\right)\right] .
\end{aligned}
$$

Поэтому согласно (3.29) находим

$$
\frac{\partial E(\mathbf{x})}{\partial t}+\frac{\partial q_{k}(\mathbf{x})}{\partial x_{k}}=-i \int d^{3} x^{\prime} \mathbf{h}^{\mathrm{eff}}\left(\mathbf{x}^{\prime}\right) \operatorname{Sp} \hat{f}\left[\widehat{\mathbf{m}}\left(\mathbf{x}^{\prime}\right), \hat{\varepsilon}(\mathbf{x}, \hat{f})\right]
$$

где

$$
q_{k}(\mathbf{x})=\frac{i}{2} \int d^{3} x^{\prime} x_{k}^{\prime} \int_{0}^{1} d \xi \operatorname{Sp} \hat{f}\left[\hat{\varepsilon}\left(\mathbf{x}-(1-\xi) \mathbf{x}^{\prime}, \hat{f}\right), \hat{\varepsilon}\left(\mathbf{x}+\xi \mathbf{x}^{\prime}, \hat{f}\right)\right] .
$$

Так как функция $\operatorname{Sp} \hat{f}[\widehat{\mathbf{m}}(\mathbf{x}), \hat{\varepsilon}(\mathbf{x}, \hat{f})]$ имеет резкий максимум при $\mathbf{x}=\mathbf{x}^{\prime}$, а функция $\mathbf{h}^{\mathrm{eff}}\left(\mathbf{x}^{\prime}\right)$ медленно меняется по переменной $\mathbf{x}^{\prime}$, правую часть равенства (3.32) можно представить в виде

$$
-i \int d^{3} x^{\prime} \mathbf{h}^{\mathrm{eff}}\left(\mathbf{x}^{\prime}\right) \operatorname{Sp} \hat{f}\left[\widehat{\mathbf{m}}\left(\mathbf{x}^{\prime}\right), \hat{\varepsilon}(\mathbf{x}, \hat{f})\right]=i \frac{\partial \mathbf{h}^{\mathrm{eff}}(\mathbf{x})}{\partial x_{k}} \operatorname{Sp} \hat{f}\left[\widehat{\mathbf{m}}(0), \hat{\varepsilon}_{k}(\hat{f})\right]
$$


где

$$
\hat{\varepsilon}_{k}(\hat{f})=\int d^{3} x x_{k} \hat{\varepsilon}(x, \hat{f})
$$

(при получении последней формулы мы предположили, что $\left[\hat{f}, \hat{p}_{k}\right]=0$, см. далее). Таким образом, уравнения (3.16)-(3.18) и (3.32) с учетом (3.22)-(3.27) и (3.34) могут быть представлены в виде дифференциальных законов сохранения с магнитными источниками

$$
\begin{gathered}
\frac{\partial \rho(\mathbf{x})}{\partial t}+\frac{\partial j_{k}(\mathbf{x})}{\partial x_{k}}=0, \quad \frac{\partial \pi_{k}(\mathbf{x})}{\partial t}+\frac{\partial t_{k l}(\mathbf{x})}{\partial x_{l}}=\mathbf{m}(\mathbf{x}) \frac{\partial \mathbf{h}^{\mathrm{eff}}(\mathbf{x})}{\partial x_{k}} \\
\frac{\partial m_{k}(\mathbf{x})}{\partial t}+\frac{\partial m_{k l}(\mathbf{x})}{\partial x_{l}}=\mu_{f}\left[\mathbf{m}(\mathbf{x}) \times \mathbf{h}^{\mathrm{eff}}(\mathbf{x})\right]_{k}, \\
\frac{\partial E(\mathbf{x})}{\partial t}+\frac{\partial q_{k}(\mathbf{x})}{\partial x_{k}}=\frac{\partial h_{l}^{\mathrm{eff}}(\mathbf{x})}{\partial x_{k}} m_{l k} .
\end{gathered}
$$

Последнее уравнение следует из того, что, как будет показано в следуюшем разделе,

$$
i \operatorname{Sp} \hat{f}\left[\widehat{m}_{l}(0), \hat{\varepsilon}_{k}(\hat{f})\right]=m_{l k} .
$$

\section{4. ПОТОКИ АДДИТИВНЫХ ИНТЕГРАЛОВ ДВИЖЕНИЯ, ОБУСЛОВЛЕННЫЕ ЯДЕРНЫМ ВЗАИМОДЕЙСТВИЕМ}

Формулы (3.25)-(3.27) и (3.33) для потоков значительно упрошаются в случае приближения малых неоднородностей. Для получения дифференциальных законов сохранения аддитивных интегралов движения в приближении, линейном по пространственным градиентам (уравнения идеальной гидродинамики), достаточно найти плотности потоков для пространственно-однородной матрицы плотности,

$$
\left[\hat{f}, p_{k}\right]=0,
$$

и считать после этого термодинамические параметры $\rho(\mathbf{x}), \pi(\mathbf{x}), \mathbf{m}(\mathbf{x})$ медленно меняющимися функциями координат и времени.

Дадим некоторое разъяснение этого утверждения. Согласно функциональной гипотезе Боголюбова [4], при временах $t$, много больших времени однородной релаксации $\tau$, статистический оператор $\hat{f}(t)$ становится универсальным функционалом гидродинамических параметров $\zeta_{\alpha}(\mathbf{x}, t)$ :

$$
\hat{f}(t) \underset{t \gg \tau}{\longrightarrow} \hat{f}\left(\zeta_{\alpha}\left(\mathbf{x}^{\prime}, t\right)\right),
$$

причем, согласно определению параметров $\zeta_{\alpha}(\mathbf{x})$,

$$
\operatorname{Sp} \hat{f}\left(\zeta_{\alpha}\left(\mathbf{x}^{\prime}\right)\right) \hat{\zeta}_{\alpha}(\mathbf{x})=\zeta_{\alpha}(\mathbf{x}) \text {. }
$$

Замечая, что $e^{-i \hat{\mathbf{p}} \mathbf{y}} \hat{\zeta}_{\alpha}(\mathbf{x}) e^{i \hat{\mathbf{p}} \mathbf{y}}=\zeta_{\alpha}(\mathbf{x}+\mathbf{y})$, из этих соотношений и кинетического уравнения (3.1) имеем

$$
e^{i \hat{\mathbf{p}} \mathbf{y}} \hat{f}\left(\hat{\zeta}_{\alpha}\left(\mathbf{x}^{\prime}\right)\right) e^{-i \hat{\mathbf{p}} \mathbf{y}}=\hat{f} \zeta_{\alpha}\left(\mathbf{x}^{\prime}+\mathbf{y}\right) .
$$

При выгислении средних $\operatorname{Sp} \hat{f}\left(\zeta_{\alpha}\left(\mathbf{x}^{\prime}\right)\right) \hat{a}(\mathbf{x})$ (где $\hat{a}(\mathbf{x})$ - оператор плотности некоторой величины $\hat{a}$, например оператор плотности потока одной из величин $\left.\zeta_{\alpha}\right)$ главный вклад в след дают те значения $\mathbf{x}^{\prime}$, которые близки к $\mathbf{x}$. Поэтому, полагая

$$
\zeta_{\alpha}\left(\mathbf{x}^{\prime}\right)=\zeta_{\alpha}(\mathbf{x})+\left(\mathbf{x}^{\prime}-\mathbf{x}\right) \frac{\partial \zeta_{\alpha}(\mathbf{x})}{\partial \mathbf{x}}+\cdots,
$$


получим разложение функционала $\hat{f}$ по градиентам параметров $\zeta_{\alpha}(\mathbf{x})$ :

$$
\begin{gathered}
\hat{f}\left(\zeta_{\alpha}\left(\mathbf{x}^{\prime}\right)\right)=\hat{f}_{0}(\mathbf{x})+\hat{f}_{1}(\mathbf{x})+\cdots \\
\hat{f}_{0}(\mathbf{x})=\hat{f}\left(\zeta_{\alpha}(\mathbf{x})\right), \quad \hat{f}_{1}(\mathbf{x})=\frac{\partial \zeta_{\alpha}(\mathbf{x})}{\partial x_{k}} \hat{g}_{\alpha k}\left(\zeta_{\beta}(\mathbf{x})\right), \quad \ldots
\end{gathered}
$$

$\left(\hat{g}_{\alpha k}-\right.$ некоторые функции $\left.\zeta_{\beta}(\mathbf{x})\right)$. Подставляя это разложение в $(4.3)$, имеем

$$
e^{i \hat{\mathbf{p}} \mathbf{y}}\left(\hat{f}_{0}(\mathbf{x})+\hat{f}_{1}(\mathbf{x})+\cdots\right) e^{-i \hat{\mathbf{p}} \mathbf{y}}=\hat{f}_{0}(\mathbf{x}+\mathbf{y})+\hat{f}_{1}(\mathbf{x}+\mathbf{y})+\cdots,
$$

откуда, дифференцируя по $\mathbf{y}$, полагая $\mathbf{y}=0$ и отбирая члены одинакового порядка по градиентам, получим

$$
i\left[\hat{p}_{k}, \hat{f}_{0}(\mathbf{x})\right]=0, \quad i\left[\hat{p}_{k}, \hat{f}_{1}(\mathbf{x})\right]=\frac{\partial \hat{f}_{0}}{\partial \zeta_{\alpha}} \frac{\partial \zeta_{\alpha}(\mathbf{x})}{\partial x_{k}}, \ldots
$$

Первое из этих соотношений доказывает наше утверждение, т.к. $\hat{f}_{0}(\mathbf{x})=\hat{f}(\zeta(\mathbf{x}))$.

Замечая, что в пространственно-однородном случае

$$
\operatorname{Sp} \hat{f}\left[\varepsilon\left(\mathbf{x}-(1-\xi) \mathbf{x}^{\prime}, \hat{f}\right), \hat{\zeta}_{\alpha}\left(\mathbf{x}+\xi \mathbf{x}^{\prime}\right)\right]=\operatorname{Sp} \hat{f}\left[\varepsilon\left(-\mathbf{x}^{\prime}, \hat{f}\right), \hat{\zeta}_{\alpha}(0)\right],
$$

имеем

$$
\begin{aligned}
j_{k} & =-i \operatorname{Sp} \hat{f}\left[\hat{\varepsilon}_{k}(\hat{f}), \hat{\rho}(0)\right], \\
t_{k l} & =-E \delta_{k l}-i \operatorname{Sp} \hat{f}\left[\hat{\varepsilon}_{l}(\hat{f}), \hat{\pi}_{k}(0)\right], \\
m_{k l} & =-i \operatorname{Sp} \hat{f}\left[\hat{\varepsilon}_{l}(\hat{f}), \hat{m}_{k}(0)\right], \\
q_{k} & =-\frac{i}{2} \operatorname{Sp} \hat{f}\left[\hat{\varepsilon}_{k}(\hat{f}), \hat{\varepsilon}(0, \hat{f})\right],
\end{aligned}
$$

где оператор $\hat{\varepsilon}_{k}(\hat{f})$ определяется формулой (3.35). Заметим, что формула (4.7) доказывает формулу (3.39). Используя (2.16), в пространственно-однородном случае имеем

$$
e^{-i \hat{\mathbf{p}} \mathbf{y}} \varepsilon(\mathbf{x}, \hat{f}) e^{i \hat{\mathbf{p}} \mathbf{y}}=\varepsilon(\mathbf{x}+\mathbf{y}, \hat{f}) .
$$

Дифференцируя это соотношение по $y_{k}$ и полагая $\mathbf{y}=0$, получаем

$$
-i\left[\hat{p}_{k}, \hat{\varepsilon}(\mathbf{x}, \hat{f})\right]=\frac{\partial \hat{\varepsilon}(\mathbf{x}, \hat{f})}{\partial x_{k}} .
$$

Отсюда следует, что

$$
i\left[\hat{p}_{k}, \hat{\varepsilon}_{l}(\hat{f})\right]=\delta_{k l} \hat{\varepsilon}(\hat{f}) .
$$

Поэтому, замечая, что матрища $\hat{\varepsilon}(\hat{f})$ единична в спиновом пространстве (в силу предположения о независимости ядерных сил от спина) и коммутирует с оператором импульса (в силу предположения о пространственной однородности), имеем

$$
i\left[\hat{f}, \hat{\varepsilon}_{l}(\hat{f})\right]=\frac{\partial \hat{f}}{\partial \hat{p}_{l}} \varepsilon(\hat{f}) .
$$


Используя выражение (4.5) для $j_{l}$, из (4.9) находим

$$
j_{l}=-\operatorname{Sp} \frac{\partial \hat{f}}{\partial \hat{p}_{l}} \varepsilon(\hat{f}) \hat{\rho}(0),
$$

или вследствие пространственной однородности

$$
j_{l}=-\frac{1}{V} \operatorname{Sp} \frac{\partial \hat{f}}{\partial \hat{p}_{l}} \hat{\varepsilon}(\hat{f}) .
$$

Интегрируя при вычислении следа по частям, получаем окончательно выражение для потока плотности квазичастиц ферми-жидкости

$$
j_{l}=\frac{1}{V} \operatorname{Sp} \hat{f} \frac{\partial \hat{\varepsilon}(\hat{f})}{\partial \hat{p}_{l}} .
$$

Используя аналогичные вычисления, находим потоки плотности импульса, магнитного момента и энергии:

$$
\begin{gathered}
t_{k l}=-\left(E-\frac{1}{V} \operatorname{Sp} \hat{f} \hat{\varepsilon}(\hat{f})\right) \delta_{k l}+\frac{1}{V} \operatorname{Sp} \hat{f} \frac{\partial \hat{\varepsilon}(\hat{f})}{\partial \hat{p}_{l}} p_{k}, \\
m_{k l}=\frac{1}{V} \operatorname{Sp} \hat{f} \frac{\partial \hat{\varepsilon}(\hat{f})}{\partial \hat{p}_{l}} \widehat{m}_{k}, \\
q_{k}=\frac{1}{V} \operatorname{Sp} \hat{f} \frac{\partial \hat{\varepsilon}(\hat{f})}{\partial \hat{p}_{k}} \hat{\varepsilon}(\hat{f}) .
\end{gathered}
$$

Эти формулы для потоков справедливы для любой пространственно-однородной матрицы плотности и имеют довольно наглядный вид, если учесть, что величина $\partial \hat{\varepsilon}(\hat{f}) / \partial \hat{p}_{k}$ является групповой скоростью квазичастиц.

Матрища плотности, входящая в (4.10)-(4.13), определяется формулой (3.8) и реализует минимум функционала $\Omega_{0}$, обусловленного только ядерным взаимодействием,

$$
\Omega_{0}=-S(\hat{f})+\beta[E(\hat{f})-\mu \operatorname{Sp} \hat{f}-\mathbf{v} \operatorname{Sp} \hat{f} \hat{\mathbf{p}}-\tilde{\mathbf{h}} \operatorname{Sp} \hat{f} \widehat{\mathbf{m}}]
$$

Вводя вместо термодинамических переменных $\beta, v_{k}(k=1,2,3), \mu$ и $\tilde{h}_{a}(a=5,6,7)$ новые термодинамические переменные

$$
Y_{0}=\beta, \quad Y_{k}=-\beta v_{k} \quad(k=1,2,3), \quad Y_{4}=-\beta \mu, \quad Y_{a}=-\beta \tilde{h}_{a} \quad(a=5,6,7),
$$

этот потенциал можно переписать в виде

$$
\Omega_{0}(\hat{f})=-S(\hat{f})+Y_{0} E(\hat{f})+Y_{k} \operatorname{Sp} \hat{f} \hat{p}_{k}+Y_{4} \operatorname{Sp} \hat{f}+Y_{a} \operatorname{Sp} \hat{f} \widehat{m}_{a} .
$$

Так как состоянию статистического равновесия, согласно ранее сказанному, соответствует минимум термодинамического потенциала $\Omega_{0}(\hat{f})$ при фиксированных $Y$, то

$$
\delta_{f} \Omega_{0}(\hat{f})=0, \quad \hat{f}=\hat{f}_{0}(Y) .
$$

Отсюда следует, что для плотности равновесного термодинамического потенциала справедливо соотношение

$$
\zeta_{\alpha}=\frac{\partial \omega(Y)}{\partial Y_{\alpha}}, \quad \alpha=0,1, \ldots, 7,
$$

5 Теоретическая и математическая физика, т. 125, № 3,2000 г. 
где $\zeta_{0}=E$ - плотность энергии, $\zeta_{k}=\pi_{k}(k=1,2,3)$ - плотность импульса, $\zeta_{4}=\rho-$ плотность числа квазичастиц и $\zeta_{a}=m_{a}(a=5,6,7)$ - плотность магнитного момента.

Найдем, наконец, плотности потоков в состоянии, описываемом матрицей плотности (3.8). Введем с этой целью функцию $f(z)=\left(e^{z}+1\right)^{-1}$, удовлетворяюшую уравнению

$$
\frac{\partial}{\partial z} \ln (1-f(z))=f(z) .
$$

Согласно (3.8)

$$
\hat{f}=f(z), \quad z=\beta[\hat{\varepsilon}(\hat{f})-\mu-\hat{\mathbf{p}} \mathbf{v}-\widehat{\mathbf{m}} \tilde{\mathbf{h}}] .
$$

Тогда из (4.10) следует

$$
j_{l}=\frac{1}{\beta V} \operatorname{Sp} \hat{f} \frac{\partial(z+\beta(\mu+\hat{\mathbf{p}} \mathbf{v}+\widehat{\mathbf{m}} \tilde{\mathbf{h}}))}{\partial p_{l}}=\frac{1}{V} v_{l} \operatorname{Sp} \hat{f}+\frac{1}{\beta V} \operatorname{Sp} \frac{\partial z}{\partial p_{k}} \frac{d}{d z} \ln (1-\hat{f}(z)) .
$$

По теореме Гаусса второе слагаемое в правой части этого выражения исчезает, и мы получаем

$$
j_{l}=n v_{l},
$$

где $n=N / V$ - плотность числа квазичастиц ферми-жидкости.

Аналогичным образом находим

$$
\operatorname{Sp} \hat{f} p_{k} \frac{\partial \varepsilon}{\partial p_{l}}=v_{l} \pi_{k}+\frac{1}{\beta V} \operatorname{Sp} p_{k} \frac{\partial z}{\partial p_{l}} \frac{d}{d z} \ln (1-\hat{f}(z))
$$

или, интегрируя по частям,

$$
\operatorname{Sp} \hat{f} p_{k} \frac{\partial \varepsilon}{\partial p_{l}}=v_{l} \pi_{k}-\delta_{l k} \frac{1}{\beta V} \operatorname{Sp} \ln (1-\hat{f}(z))
$$

и, следовательно,

$$
t_{k l}=v_{l} \pi_{k}+\delta_{l k}\left[\frac{1}{V} \operatorname{Sp} \hat{f} \hat{\varepsilon}(\hat{f})-E-\frac{1}{\beta V} \operatorname{Sp} \ln (1-\hat{f}(z))\right] .
$$

Подобным же образом нетрудно показать, что

$$
\begin{gathered}
q_{l}=v_{l}\left(\frac{1}{V} \operatorname{Sp} \hat{f} \hat{\varepsilon}(\hat{f})-\frac{1}{\beta V} \operatorname{Sp} \ln (1-\hat{f}(z))\right), \\
m_{k l}=m_{k} v_{l} .
\end{gathered}
$$

С другой стороны, из (4.14) вытекает, что потенщиал $\omega=\Omega_{0} / V$ можно представить в виде

$$
\omega=\frac{1}{V} \operatorname{Sp} \ln (1-\hat{f})+\beta\left(E-\frac{1}{V} \operatorname{Sp} \hat{f} \hat{\varepsilon}(\hat{f})\right) .
$$

Поэтому формулы (4.21), (4.24)-(4.26) можно переписать в виде

$$
j_{l}=n v_{l}, \quad t_{l k}=v_{k} \pi_{l}-\frac{\omega}{\beta} \delta_{l k}, \quad q_{l}=v_{l}\left(E-\frac{\omega}{\beta}\right), \quad m_{k l}=m_{k} v_{l},
$$

так что величину $-\omega / \beta=P$ следует интерпретировать как давление, а величину $E-$ $\omega / \beta$ - как плотность энтальпии. 
Из (4.28) и (4.18) следует, что потоки физических величин $\zeta_{\alpha k}$ можно представить в виде

$$
\zeta_{\alpha k}=-\frac{\partial}{\partial Y_{\alpha}} \frac{\omega Y_{k}}{Y_{0}}
$$

где $\zeta_{0 k}=q_{k}, \zeta_{l k}=t_{l k}, \zeta_{4 k}=j_{k}, \zeta_{a k}=m_{a k}$. Поэтому уравнения гидродинамики нейтронной материи имеют вид

$$
\frac{\partial \zeta_{\alpha}}{\partial t}+\frac{\partial \zeta_{\alpha k}}{\partial x_{k}}=X_{\alpha}, \quad \alpha=0,1, \ldots, 7,
$$

где

$$
X_{0}=\frac{\partial h_{i}^{\mathrm{eff}}}{\partial x_{k}} m_{i} v_{k}, \quad X_{k}=\frac{\partial h_{i}^{\mathrm{eff}}}{\partial x_{k}} m_{i}, \quad X_{4}=0, \quad X_{a}=\mu_{f}\left[\mathbf{m} \times \mathbf{h}^{\mathrm{eff}}\right]_{a},
$$

причем в согласии с принципом локального статистического равновесия величины $\zeta_{\alpha}$ и $\zeta_{\alpha k}$ зависят от $\mathbf{x}$ и $t$ только посредством медленно меняюшихся параметров $Y_{\alpha}(\mathbf{x}, t)(\mathrm{cm}$. (4.18))

\section{5. ИНВАРИАНТНОСТЬ ФУНКЩИОНАЛА ЭНЕРГИИ ОТНОСИТЕЛЬНО ПРЕОБРАЗОВАНИЙ ГАЛИЛЕЯ}

Будем говорить, что ферми-жидкость обладает свойством галилеевой инвариантности, если функционал плотности энергии удовлетворяет соотношению

$$
E\left(\mathbf{x}, U_{u} \hat{f} U_{u}^{+}\right)=E(\mathbf{x}, \hat{f})+u_{i} \pi_{i}(\mathbf{x}, \hat{f})+\frac{1}{2} m u^{2} \rho(\mathbf{x}, \hat{f}),
$$

где

$$
\pi_{i}(\mathbf{x}, \hat{f})=\operatorname{Sp} \hat{f} \hat{\pi}_{i}(\mathbf{x}), \quad \rho_{i}(\mathbf{x}, \hat{f})=\operatorname{Sp} \hat{f} \hat{\rho}_{i}(\mathbf{x})
$$

и

$$
U_{u}=e^{i m \mathbf{u} \hat{\mathbf{x}}}
$$

( $m$ - масса фермиона).

Если плотность энергии представить в виде

$$
E(\mathbf{x}, \hat{f})=E_{0}(\mathbf{x}, \hat{f})+E_{\text {int }}(\mathbf{x}, \hat{f}),
$$

где

и учесть, что

$$
E_{0}(\mathbf{x}, \hat{f})=\frac{1}{2 m} \operatorname{Sp} \hat{f} \hat{\mathbf{p}} \delta(\mathbf{x}-\hat{\mathbf{x}}) \hat{\mathbf{p}}
$$

$$
E_{0}\left(\mathbf{x}, U_{u} \hat{f} U_{u}^{+}\right)=E_{0}(\mathbf{x}, \hat{f})+u_{i} \pi_{i}(\mathbf{x}, \hat{f})+\frac{1}{2} m u^{2} \rho(\mathbf{x}, \hat{f}),
$$

то соотношение (5.1) можно переписать как

$$
E_{\text {int }}\left(\mathbf{x}, U_{u} \hat{f} U_{u}^{+}\right)=E_{\text {int }}(\mathbf{x}, \hat{f}) .
$$

Из определения (2.11) плотности энергии квазичастицы и соотношения (5.1) следует, что

$$
U_{u}^{+} \hat{\varepsilon}(\mathbf{x}, \hat{f}) U_{u}=\hat{\varepsilon}\left(\mathbf{x}, U_{u}^{+} \hat{f} U_{u}\right)+u_{i} \hat{\pi}_{i}(\mathbf{x}, \hat{f})+\frac{1}{2} m u^{2} \hat{\rho}(\mathbf{x}, \hat{f}) .
$$


Эта формула показывает, что для квазиравновесной матрицы плотности (3.8) справедливо соотношение

$$
U_{v}^{+} \hat{f} U_{v}=\left\{\exp \left[\hat{\varepsilon}\left(U_{v}^{+} \hat{f} U_{v}\right)-\mu-\frac{1}{2} m v^{2}-\widehat{m}_{i} \tilde{h}_{i}\right]+1\right\}^{-1},
$$

из которого следует, что если ввести обозначение

$$
\hat{f}=\hat{f}(Y),
$$

TO

$$
U_{v}^{+} \hat{f}(Y) U_{v}=\hat{f}\left(Y^{0}\right)
$$

где термодинамические силы $Y^{0}$ определяются формулами

$$
Y_{0}^{0}=Y_{0}, \quad Y_{k}^{0}=0, \quad Y_{4}^{0}=Y_{4}-\frac{1}{2} m v^{2} Y_{0}, \quad Y_{a}^{0}=Y_{a} .
$$

Эти формулы показывают, что среднее значение произвольной физической величины $\hat{a}$ в состоянии $Y$ можно представить в виде

$$
\operatorname{Sp} \hat{f}(Y) \hat{a}=\operatorname{Sp} \hat{f}\left(Y^{0}\right) U_{v}^{+} \hat{a} U_{v} .
$$

Замечая, что

$$
U_{v}^{+} \hat{\rho}(\mathbf{x}) U_{v}=\hat{\rho}(\mathbf{x}), \quad U_{v}^{+} \hat{\pi}_{i}(\mathbf{x}) U_{v}=\hat{\pi}_{i}(\mathbf{x})+m v_{i} \hat{\rho}(\mathbf{x}), \quad U_{v}^{+} \widehat{m}_{i}(\mathbf{x}) U_{v}=\widehat{m}_{i}(\mathbf{x}),
$$

и учитывая, что $\operatorname{Sp} \hat{f}\left(Y^{0}\right) \hat{\pi}_{i}(\mathbf{x})=0$, получим отсюда

$$
\rho(Y)=\rho\left(Y^{0}\right), \quad \pi_{i}(Y)=m v_{i} \rho\left(Y^{0}\right), \quad m_{i}(Y)=m_{i}\left(Y^{0}\right) .
$$

Сравнение этих формул с выражением (4.21) для $j_{i}$ показывает, что в галилеевоинвариантной теории имеет место соотношение

$$
\pi_{i}(Y)=m j_{i}(Y) .
$$

Подчеркнем, что только в галилеево-инвариантной теории есть понятие массы фермиона $m$, причем эта величина вводится в теорию с помошью соотношения (5.1).

Преобразуем, наконец, выражение для плотности энергии $E(Y)$ в состоянии статистического равновесия. Согласно (5.1) имеем

$$
E(Y)=E\left(\mathbf{x}, U_{u}^{+} \hat{f} U_{u}\right)+u_{i} \pi_{i}\left(\mathbf{x}, U_{u}^{+} \hat{f} U_{u}\right)+\frac{1}{2} m u^{2} \rho\left(\mathbf{x}, U_{u}^{+} \hat{f} U_{u}\right)
$$

или, учитьвая (5.11), имеем при $\mathbf{u}=\mathbf{v}$

$$
E(Y)=E\left(Y^{0}\right)+v_{i} \operatorname{Sp} \hat{f}\left(Y^{0}\right) \hat{\pi}_{i}(\mathbf{x})+\frac{1}{2} m v^{2} \operatorname{Sp} \hat{f}\left(Y^{0}\right) \hat{\rho}(\mathbf{x}),
$$

и, следовательно,

$$
E(Y)=E_{0}+\frac{1}{2} m v^{2} \rho\left(Y^{0}\right)
$$

где $E_{0}=E\left(Y^{0}\right)$. 


\section{6. ДИФФЕРЕНЦИАЛЬНЫЕ ЗАКОНЫ СОХРАНЕНИЯ}

В отсутствие внешнего магнитного поля функционал энергии (3.9) инвариантен относительно преобразований пространственно-временных трансляций и пространственных (с учетом спина) поворотов. Поэтому в этом случае уравнения магнитной гидродинамики можно привести к виду дифференциальных законов сохранения.

Уравнение (4.30) при $\alpha=4$ уже имеет вид дифференциального закона сохранения

$$
\frac{\partial \rho(\mathbf{x})}{\partial t}+\frac{\partial j_{k}(\mathbf{x})}{\partial x_{k}}=0, \quad j_{k}=\rho v_{k},
$$

где $v_{k}=-Y_{k} / Y_{0}$.

Уравнение (4.30) при $\alpha=i=1,2,3$ принимает вид дифференциального закона сохранения для плотности импульса $\pi_{i}=m \rho v_{i}$ (при учете галилеевой инвариантности, см. раздел 5)

$$
\begin{gathered}
\frac{\partial \rho v_{i}}{\partial t}+\frac{\partial}{\partial x_{k}} \Pi_{i k}=0 \\
\Pi_{i k}=m \rho v_{i} v_{k}+P \delta_{i k}-\frac{1}{4 \pi}\left(h_{i} b_{k}-\frac{1}{2} \mathbf{h}^{2} \delta_{i k}\right)-\alpha_{k s} \mathbf{m} \frac{\partial^{2} \mathbf{m}}{\partial x_{s} \partial x_{i}}+\frac{1}{2} \delta_{i k} \alpha_{l s} \frac{\partial \mathbf{m}}{\partial x_{l}} \frac{\partial \mathbf{m}}{\partial x_{s}},
\end{gathered}
$$

где

$$
P=-\frac{\omega}{\beta}+\mathbf{m}^{2} R^{\prime}-\frac{1}{2} R
$$

Мы учли при этом, что

$$
\begin{gathered}
\mathbf{m} \frac{\partial \mathbf{h}}{\partial x_{i}}=\frac{\partial}{\partial x_{k}} \frac{1}{4 \pi}\left(h_{i} b_{k}-\frac{1}{2} \mathbf{h}^{2} \delta_{i k}\right), \\
\mathbf{m} \frac{\partial}{\partial x_{i}} \alpha_{l s} \frac{\partial^{2} \mathbf{m}}{\partial x_{l} \partial x_{s}}=\frac{\partial}{\partial x_{k}}\left(\alpha_{k s} \mathbf{m} \frac{\partial^{2} \mathbf{m}}{\partial x_{s} \partial x_{i}}-\frac{1}{2} \delta_{i k} \alpha_{l s} \frac{\partial \mathbf{m}}{\partial x_{l}} \frac{\partial \mathbf{m}}{\partial x_{s}}\right) .
\end{gathered}
$$

Уравнение (4.30) при $\alpha=a=5,6,7$ может быть записано в виде

$$
\frac{\partial m_{i}}{\partial t}+\operatorname{div} m_{i} \mathbf{v}=\mu_{f}\left[\mathbf{m} \times \mathbf{h}^{\mathrm{eff}}\right]_{i} .
$$

Для того чтобы придать этому уравнению вид дифференциального закона сохранения, введем плотность полного момента количества движения

$$
J_{i}=\varepsilon_{i k l} x_{k} \pi_{l}+\frac{1}{\mu_{f}} m_{i}
$$

Тогда согласно (6.2) и (6.4) имеем

$$
\begin{gathered}
\frac{\partial J_{i}}{\partial t}+\frac{\partial J_{i k}}{\partial x_{k}}=0 \\
J_{i k}=\varepsilon_{i p l} x_{p} \Pi_{l k}+\frac{1}{\mu_{f}} m_{i} v_{k}-\varepsilon_{i p l} m_{p} \alpha_{k r} \frac{\partial m_{l}}{\partial x_{r}} .
\end{gathered}
$$

Мы учли при этом, что (см. (3.14), (6.3))

$$
\varepsilon_{i s l} \Pi_{l s}+\varepsilon_{i s l} m_{s} h_{l}^{\mathrm{eff}}=\frac{\partial}{\partial x_{p}} \varepsilon_{i s l} m_{s} \alpha_{p r} \frac{\partial m_{l}}{\partial x_{r}} .
$$


Получим, наконец, дифференциальный закон сохранения энергии. С этой целью заметим, что

$$
\begin{aligned}
\frac{\partial}{\partial t}\left(\frac{\mathbf{h}^{2}}{8 \pi}\right. & \left.+\frac{1}{2} \alpha_{i k} \frac{\partial \mathbf{m}}{\partial x_{i}} \frac{\partial \mathbf{m}}{\partial x_{k}}+\frac{1}{2} R\right)= \\
= & \frac{1}{4 \pi} \frac{\partial}{\partial x_{k}}[\mathbf{e} \times \mathbf{h}]_{k}-\mathbf{h}^{\mathrm{eff}} \frac{\partial \mathbf{m}}{\partial t}+\frac{\partial}{\partial x_{k}}\left(\alpha_{i k} \frac{\partial \mathbf{m}}{\partial x_{i}} \frac{\partial \mathbf{m}}{\partial t}\right)
\end{aligned}
$$

где учтено, что

$$
\frac{\partial \mathbf{b}}{\partial t}=-\operatorname{rot} \mathbf{e}
$$

и использовано определение эффективного поля $\mathbf{h}^{\mathrm{eff}}$. Вводя полную плотность энергии

$$
E_{t}=E+\frac{h^{2}}{8 \pi}+\frac{1}{2} \alpha_{i k} \frac{\partial \mathbf{m}}{\partial x_{i}} \frac{\partial \mathbf{m}}{\partial x_{k}}+\frac{1}{2} R
$$

и используя уравнение (4.30) при $\alpha=0$, получим окончательно

$$
\begin{gathered}
\frac{\partial E_{t}}{\partial t}+\frac{\partial \Pi_{k}}{\partial x_{k}}=0 \\
\Pi_{k}=v_{k}\left(E+P+\frac{1}{2} R-\mathbf{m}^{2} R^{\prime}\right)+\frac{1}{8 \pi}[\mathbf{e} \times \mathbf{h}]_{k}-\mathbf{m h}^{\text {eff }} v_{k}-\alpha_{i k} \frac{\partial \mathbf{m}}{\partial x_{i}} \frac{\partial \mathbf{m}}{\partial t} .
\end{gathered}
$$

В нулевом приближении по градиентам формулы для плотностей потоков принимают Вид

$$
\begin{gathered}
j_{k}=\rho v_{k}, \quad \Pi_{i k}=m \rho v_{i} v_{k}+P \delta_{i k}-\frac{1}{4 \pi}\left(h_{i} b_{k}-\frac{1}{2} \mathbf{h}^{2} \delta_{i k}\right), \\
J_{i k}=\varepsilon_{i p l} x_{p} \Pi_{l k}+\frac{1}{\mu_{f}} m_{i} v_{k}, \quad \Pi_{k}=v_{k}\left(\frac{m \rho v^{2}}{2}+w_{0}+P\right)+\frac{1}{8 \pi}[\mathbf{e} \times \mathbf{h}]_{k}-\mathbf{m h} v_{k},
\end{gathered}
$$

где $w_{0}=E_{0}+R / 2$.

Вместо закона сохранения энергии часто используют закон адиабатичности для плотности энтропии $\sigma$. Согласно (4.16) $\omega=-\sigma+Y_{a} \zeta_{a}$, причем в соответствии с (4.18)

$$
d \omega=\zeta_{a} d Y_{a},
$$

и, следовательно,

$$
d \sigma=Y_{a} d \zeta_{a}
$$

Таким образом, согласно (4.30)

$$
\frac{\partial \sigma}{\partial t}=Y_{a} \frac{\partial \zeta_{a}}{\partial t}=Y_{a}\left(-\frac{\partial \zeta_{a k}}{\partial x_{k}}+X_{a}\right)
$$

или

$$
\frac{\partial \sigma}{\partial t}=-\frac{\partial}{\partial x_{k}}\left(Y_{a} \zeta_{a k}\right)+\zeta_{a k} \frac{\partial Y_{a}}{\partial x_{k}}+Y_{a} X_{a}
$$


Так как

$$
\zeta_{a k}=-\frac{\partial}{\partial Y_{a}} \frac{\omega Y_{k}}{Y_{0}}
$$

TO

$$
\frac{\partial \sigma}{\partial t}=-\frac{\partial}{\partial x_{k}}\left(Y_{a} \zeta_{a k}+\frac{\omega Y_{k}}{Y_{0}}\right)+Y_{a} X_{a}
$$

Замечая, что

$$
Y_{a} \zeta_{a k}=-Y_{a} \frac{\partial}{\partial Y_{a}} \frac{\omega Y_{k}}{Y_{0}} \text { и } Y_{a} \frac{\partial}{\partial Y_{a}} \frac{Y_{k}}{Y_{0}}=0
$$

имеем

$$
\frac{\partial \sigma}{\partial t}=-\frac{\partial}{\partial x_{k}} \frac{Y_{k}}{Y_{0}}\left(\omega-Y_{a} \zeta_{a}\right)+Y_{a} X_{a}=-\frac{\partial}{\partial x_{k}} v_{k} \sigma+Y_{a} X_{a}
$$

С другой стороны, в соответствии с (4.31)

$$
Y_{a} X_{a}=Y_{0} \frac{\partial h_{i}^{\mathrm{eff}}}{\partial x_{k}} m_{i} v_{k}+Y_{k} m_{i} \frac{\partial h_{i}^{\mathrm{eff}}}{\partial x_{k}}+\mu_{f} \mathbf{Y}\left[\mathbf{h}^{\mathrm{eff}} \times \mathbf{m}\right]=0,
$$

т.к. согласно (4.18) вектор $\mathbf{Y}$ параллелен $\mathbf{m}$ (термодинамический потенциал $\omega$ зависит от квадрата вектора $\mathbf{Y})$.

Таким образом, имеем окончательно

$$
\frac{\partial \sigma}{\partial t}+\operatorname{div} \sigma \mathbf{v}=0
$$

Вектор $\sigma \mathbf{v}$ следует интерпретировать как плотность потока энтропии.

\section{7. УДАРНЫЕ ВОЛНЫ В НЕЙТРОННОЙ МАТЕРИИ}

В качестве приложения полученных уравнений рассмотрим волны большой амплитуды, которые могут распространяться в нейтронной материи, - ударные волны [1]. Будем для простоты вначале считать течение одномерным, т.е. считать, что все величинь зависят только от одной координаты $x$. Обозначим через $a_{\alpha}(x)$ плотность физических величин

$$
a_{\alpha}(x)=\rho(x), \quad \rho(x) v_{i}(x), \quad[\mathbf{r}, \rho \mathbf{v}]_{i}+\frac{1}{\mu_{f}} m_{i},
$$

а через $b_{\alpha k}(x)$ - соответствуюшие плотности потоков, которые являются функциями плотностей $a_{\alpha}(x), b_{\alpha k}(x)=b_{\alpha k}\left(a_{\alpha}(x)\right)$. Тогда дифференциальные законы сохранения $(5.1),(5.2),(5.6)$ и (5.12) могут быть представлены в виде

$$
\dot{a}_{\alpha}+\frac{\partial b_{\alpha k}}{\partial x_{k}}=0
$$

или в одномерном случае

$$
\dot{a}_{\alpha}+\frac{\partial b_{\alpha}}{\partial x}=0, \quad b_{\alpha}(x)=b_{\alpha}\left(a_{\beta}(x)\right),
$$

где $b_{\alpha}=b_{\alpha x}$. 
Чтобы описать разрывные течения, положим

$$
a_{\alpha}(x)=a_{\alpha}^{+}(x) \theta(x-x(t))+a_{\alpha}^{-}(x) \theta(-x+x(t)),
$$

где $x(t)-x$-координата поверхности разрыва в момент времени $t$,

$$
\theta(x)= \begin{cases}1, & x \geq 0 \\ 0, & x<0\end{cases}
$$

В соответствии с этим величины $b_{\alpha}$ представятся в виде

$$
b_{\alpha}(x)=b_{\alpha}^{+}(x) \theta(x-x(t))+b_{\alpha}^{-}(x) \theta(-x+x(t)),
$$

где $b_{\alpha}^{ \pm}(x)=b_{\alpha}\left(a_{\alpha}^{ \pm}\right)$.

Подставляя (7.3) и (7.4) в уравнение (7.2) и замечая, что

$$
\frac{\partial}{\partial t} \theta(x-x(t))=-\dot{x}(t) \delta(x-x(t)),
$$

получим

$$
\begin{aligned}
\left(\dot{a}_{\alpha}^{+}+\right. & \left.\frac{\partial b_{\alpha}^{+}}{\partial x}\right) \theta(x-x(t))+\left(\dot{a}_{\alpha}^{-}+\frac{\partial b_{\alpha}^{-}}{\partial x}\right) \theta(-x+x(t))+ \\
& +\delta(x-x(t))\left(-\dot{x}(t)\left(a_{\alpha}^{+}-a_{\alpha}^{-}\right)+b_{\alpha}^{+}-b_{\alpha}^{-}\right)=0 .
\end{aligned}
$$

Отсюда находим уравнения магнитной гидродинамики слева и справа от поверхности разрыва

$$
\dot{a}_{\alpha}^{ \pm}+\frac{\partial b_{\alpha}^{ \pm}}{\partial x}=0
$$

и граничные условия на поверхности разрьва

$$
\left\{b_{\alpha}\right\}=\dot{x}(t)\left\{a_{\alpha}\right\}
$$

где через $\{\ldots\}$ обозначена разность значений физических величин слева и справа от поверхности разрыва. Например,

$$
\left\{a_{\alpha}\right\}=\left.\left(a_{\alpha}^{+}-a_{\alpha}^{-}\right)\right|_{x=x(t)} .
$$

Представляя уравнения Максвелла в виде, аналогичном формуле (7.6), найдем граничные условия для электромагнитных полей на поверхности разрыва

$$
\left\{\varepsilon_{i 1 k} e_{k}\right\}=\frac{\dot{x}(t)}{c}\left\{b_{i}\right\}, \quad\left\{\varepsilon_{i 1 k} h_{k}\right\}=0, \quad\left\{b_{1}\right\}=0 .
$$

Эти условия можно переписать в виде

$$
\left\{\left(\mathbf{e}+\frac{1}{c}[\mathbf{u}, \mathbf{b}]\right)_{t}\right\}=0, \quad\left\{\mathbf{h}_{t}\right\}=0, \quad\left\{\mathbf{b}_{n}\right\}=0,
$$

где индексом $t$ обозначены тангенциальные составляющие полей, а индексом $n$ - нормальные составляющие. Причем $\mathbf{u}(t)=\dot{\mathbf{x}}(t)$ - нормальная составляющая скорости движения поверхности разрыва. В такой форме эти граничные условия справедливы для 
любого течения, не обязательно одномерного. Аналогичным образом формулы (7.7) могут быть записаны для произвольного разрывного течения

$$
\left\{b_{\alpha k} n_{k}\right\}=u(t)\left\{a_{\alpha}\right\}, \quad u(t)=u_{k}(t) n_{k}
$$

( $\mathbf{n}$ - единичный вектор нормали к поверхности разрыва). В случае плоской поверхности разрыва, которая движется равномерно со скоростью $u$, уравнения магнитной гидродинамики допускают решения

$$
a_{\alpha}^{+}=\text {const }, \quad a_{\alpha}^{-}=\text {const },
$$

причем указанные константы связаны между собой соотношениями

$$
b_{\alpha}\left(a^{+}\right)-b_{\alpha}\left(a^{-}\right)=u\left(a_{\alpha}^{+}-a_{\alpha}^{-}\right) .
$$

Уравнения (7.9) справедливы в произвольной системе отсчета. Мы рассмотрим эти уравнения в системе отсчета, где разрыв покоится, $u=0$,

$$
\left\{\rho v_{k} n_{k}\right\}=0, \quad\left\{\pi_{i k} n_{k}\right\}=0, \quad\left\{\Pi_{k} n_{k}\right\}=0, \quad\left\{J_{i k} n_{k}\right\}=0 .
$$

Именно в таком виде граничные условия на поверхности разрыва обычно и используются $[5,6]$.

K этим соотношениям должны быть добавлены уравнения для скачков электромагнитных полей

$$
\left\{b_{k} n_{k}\right\}=0, \quad \varepsilon_{i k l}\left\{h_{k} n_{l}\right\}=0, \quad \varepsilon_{i k l}\left\{e_{k} n_{l}\right\}=0 .
$$

Используя определения (5.7) и (5.13) плотностей потоков и учитывая галилееву инвариантность, представим уравнения (7.10) и (7.11) в виде

$$
\begin{gathered}
\left\{\rho m v_{n}\left(\frac{v_{n}^{2}}{2}+\frac{v_{t}^{2}}{2}\right)+\rho m v_{n}\left(\frac{P+w_{0}}{\rho}\right)-\mathbf{h m} v_{n}\right\}=0, \\
\left\{P+\rho m v_{n}^{2}-\frac{h_{n}^{2}}{8 \pi}-h_{n} m_{n}\right\}=0, \\
\left\{\rho m \mathbf{v}_{t} v_{n}-\frac{1}{4 \pi} \mathbf{h}_{t} b_{n}\right\}=0, \quad\left\{v_{n} \mathbf{m}\right\}=0 .
\end{gathered}
$$

При получении второго из этих уравнений мы учли, что $\left\{h_{t}^{2}\right\}=0$. Учитывая первое из уравнений (7.11), представим третье из уравнений (7.12) в виде $\left\{\rho \mathbf{v}_{t} v_{n}\right\}=0$.

Под ударными волнами понимаются такие волны, в которых на поверхности разрыва плотность $\rho$ испытывает скачок [6]. Обозначая через $j^{m}$ нормальную составляюшую плотности потока вешества $j^{m}=m j=\rho m v_{n}$ (она непрерывна на поверхности разрыва, $j \neq 0)$ и вводя обозначение $V=1 /(\rho m)$ ( $V$ - удельный объем), перепишем уравнения (7.12) в виде

$$
\begin{gathered}
\left\{\left(P+w_{0}\right) V+\frac{\left(j^{m}\right)^{2} V^{2}}{2}-V \mathbf{h m}\right\}=0, \\
\left\{P+\left(j^{m}\right)^{2} V-\frac{h_{n}^{2}}{8 \pi}-h_{n} m_{n}\right\}=0, \\
\left\{\mathbf{v}_{t}\right\}=0, \quad\{V \mathbf{m}\}=0 .
\end{gathered}
$$


Исключая из первых двух уравнений величину $\left(j^{m}\right)^{2}$, получим

$$
\left\{\left(P+w_{0}\right) V\right\}-\frac{V_{1}+V_{2}}{2}\{P\}+\frac{V_{1}+V_{2}}{2} \frac{\left\{h_{n}^{2}\right\}}{8 \pi}+\frac{V_{1}+V_{2}}{2}\left\{h_{n} m_{n}\right\}-\{V \mathbf{h m}\}=0 .
$$

Используя (7.11), запишем последнее уравнение в виде

$$
\left(\varepsilon_{1}-\varepsilon_{2}\right)+\frac{P_{1}+P_{2}}{2}\left(V_{1}-V_{2}\right)+\pi\left(V_{1}-V_{2}\right)\left(m_{n 1}-m_{n 2}\right)^{2}=0,
$$

где $\varepsilon=w_{0} V$ - энергия единищы массы среды. Это уравнение представляет собой обобшение уравнения адиабаты Гюгонио для ударных волн в нейтронной материи. Оно отличается от уравнения адиабаты Гюгонио в обычной гидродинамике [6] тем, что имеется дополнительное слагаемое

$$
\pi\left(V_{1}-V_{2}\right)\left(m_{n 1}-m_{n 2}\right)^{2}=\frac{1}{16 \pi}\left(V_{1}-V_{2}\right)\left(h_{n 1}-h_{n 2}\right)^{2} .
$$

В свою очередь, уравнение (7.13) отличается от уравнения ударной адиабаты в обычной магнитной гидродинамике [5] тем, что $\left(\mathbf{h}_{t 1}-\mathbf{h}_{t 2}\right)^{2}$ заменяется, как это видно из $(7.14)$, на $\left(h_{n 1}-h_{n 2}\right)^{2}$.

В заключение вьпишем выражения для скачков скорости и квадрата нормальной составляющей магнитного момента

$$
\begin{gathered}
\left(v_{1}-v_{2}\right)=\sqrt{\left(P_{2}^{*}-P_{1}^{*}\right)\left(V_{1}-V_{2}\right)}, \\
2 \pi\left(V_{1}-V_{2}\right)\left(m_{n 1}-m_{n 2}\right)^{2}=\frac{\left(v_{1}-v_{2}\right)^{2}}{V_{1}-V_{2}}-\left(P_{2}^{*}-P_{1}^{*}\right)=0,
\end{gathered}
$$

где $P^{*}=P+2 \pi m_{n}^{2}$.

Скачок магнитного момента может двигаться с произвольной скоростью. Наличие движушегося скачка магнитного момента приводит к изменению в пространстве и во времени магнитного момента среды, что, в свою очередь, приводит к излучению электромагнитных волн. Иными словами, распространение ударной волны должно сопровождаться излучением электромагнитных волн. Мы, однако, в этой статье данный вопрос изучать не будем.

Благодарности. Работа выполнена при финансовой поддержке Украинского научно-технического центра (проект 1499).

\section{Список литературы}

[1] A. I. Akhiezer, N. V. Laskin, S. V. Peletminskii. Phys. Rev. E. 1996. V. 58. № 5. P. 6512-6516.

[2] Л. Д. Ландау. ЖЭТФ. 1957. Т. 32. С. 59; Собрание трудов. Т. 2. М.: Наука, 1969.

[3] А.И. Ахиезер, В. Г. Барълхтар, С. В. Пелетминский. Спиновые волны. М.: Наука, 1967.

[4] Н. Н. Боголюбов. Проблемы динамической теории в статистической физике. М.-Л.: ОГИЗ, Гостехиздат, 1946; Избраные труды в 3-х томах. Т. 2. Киев: Наукова думка, 1970.

[5] Л. Д. Ландау, Е. М. Лифиии. Электродинамика сплошных сред. М.: Наука, 1992.

[6] Л.Д. Ландау, Е. М. Лифииц. Механика сплошных сред. М.: ГТТИ, 1945. 